\title{
O Programa Nacional de Cooperação Acadêmica (Procad) na visão dos avaliadores de projetos
}

\section{The National Program of Academic Cooperation (PROCAD) in the view of the project evaluators}

\section{El Programa Nacional de Cooperación Académica (PROCAD) en la visión de los evaluadores de proyectos}

Elci Vieira de Moura, doutoranda em Educação em Ciências pela Universidade Federal do Rio Grande do Sul (UFRGS) e coordenadora de Programas Especiais Substituta da Coordenação de Aperfeiçoamento de Pessoal de Nível Superior (Capes), Brasília, DF, Brasil. E-mail: elci.moura@capes.gov.br.

Ivan Rocha Neto, doutor em Eletrônica pela University of Kent, Canterbury, Reino Unido, e professor colaborador da Universidade Federal do Rio Grande do Sul (UFRGS), Brasília, DF, Brasil. E-mail: neto-ivan@hotmail.com.

\section{Resumo}

Este artigo teve como propósito apresentar e analisar as percepções, as críticas e as sugestões dos consultores que participaram de processos avaliativos no âmbito do Programa Nacional de Cooperação Acadêmica (Procad), tanto na submissão de propostas quanto na avaliação intermediária dos projetos em andamento, visando contribuir para o aprimoramento do programa. A pesquisa de natureza qualitativa e quantitativa foi realizada mediante consulta, por correio eletrônico, enviada aos integrantes da comissão de avaliação de projetos Procad. O questionário foi composto por 15 quesitos de natureza qualitativa, abrangendo 13 questões fechadas e duas abertas, relativas aos objetivos e às características do programa. Com base neste estudo, foi possível compreender que a institucionalização de um sistema de avaliação contínua para o Procad poderia contribuir para o aprimoramento do 
programa e para a criação de novas ações voltadas ao desenvolvimento da pós-graduação. A implantação de medidas aqui sugeridas também proporcionaria melhorias ao Procad.

Palavras-chave: Avaliação. Capes. Cooperação Acadêmica. Procad.

\section{Abstract}

This article had the purpose of presenting and analyzing the perceptions, criticisms and suggestions of the consultants who participated of the evaluation processes of the National Program of Academic Cooperation (PROCAD), considering both the submission of proposals and the mid-term evaluation of ongoing projects in an effort to contribute to the improvement of the program. The qualitative and quantitative research was carried out through consultation by email, sent to members of the Evaluation Committee of PROCAD projects. The questionnaire consisted of 15 items of a qualitative nature, covering 13 closed and two open questions relating to the aims and characteristics of the program. Based on this study, it can be understood that the institutionalization of a system of ongoing evaluation for PROCAD could contribute to the improvement of the program and to the creation of new initiatives aimed at developing graduate study. The implementation of the measures suggested here would also provide for the improvement to PROCAD.

Keywords: Assessment. CAPES. Academic Cooperation. PROCAD.

\section{Resumen}

Este artículo tuvo como objetivo presentar y analizar las percepciones, críticas y sugerencias de los consultores que participaron en los procesos de evaluación en el marco del Programa Nacional de Cooperación Académica (PROCAD), en la presentación de propuestas y en la evaluación intermedia de los proyectos en curso, con el objetivo de contribuir a la mejora del programa. La investigación cualitativa 
y cuantitativa se llevó a cabo a través de un cuestionario por correo electrónico, enviado a los miembros del Comité de Evaluación de proyectos PROCAD. El cuestionario constaba de 15 preguntas de carácter cualitativo, que cubrieron 13 preguntas cerradas y dos abiertas relacionadas con los objetivos y características del Programa. Sobre la base de este estudio, se puede entender que la institucionalización de un sistema de evaluación continua para el PROCAD podría contribuir a la mejora del programa y la creación de nuevas acciones destinadas a desarrollar la posgrado. La aplicación de las medidas que aquí se sugiere también proporcionaría mejoras para el PROCAD.

Palabras clave: Evaluación. CAPES. Cooperación Académica. PROCAD.

\section{Introdução}

A partir da análise dos Planos Nacionais de Pós-Graduação - I PNPG (1975-1979), II PNPG (1982-1985) e III PNPG (1986-1989) -, é possivel constatar que a política de pós-graduação no Brasil teve inicialmente o objetivo de capacitar os docentes das universidades. Posteriormente, houve preocupação com o desempenho do sistema de pós-graduação e, finalmente, ganhou destaque o desenvolvimento da pesquisa na universidade, como forma de impulsionar a pesquisa científica e tecnológica e atender às prioridades nacionais. Entretanto, a preocupação com as disparidades regionais na pós-graduação não deixou de ser enfatizada pela referida política (BRASIL, 2005c).

Apesar de o IV PNPG, que estava programado para ser divulgado em 1996, não ter sido oficialmente lançado, várias de suas recomendações foram implantadas pela Coordenação de Aperfeiçoamento de Pessoal de Nivel Superior (Capes), entre elas, ações visando à redução do desequilíbrio regional (BRASIL, 2005c). Nesse sentido, na percepção de Guimarães (2009), a Capes prioriza ações que têm por objetivo a redução das assimetrias regionais na pós-graduação, sendo esse o principal tema do Plano Nacional de Pós-Graduação 2005-2010. Ele enfatiza ainda que tais ações visam à qualificação de doutores e à consequente criação de programas de pós-graduação, especialmente nas regiões 
Norte, Nordeste e Centro-Oeste, exceto o Distrito Federal, considerando a menor concentração de pessoal qualificado em relação às demais regiões brasileiras.

Nesse contexto, o PNPG 2005-2010 definiu como um dos principais desafios enfrentados pelo país a necessidade de redução das disparidades regionais e estaduais na pós-graduação brasileira. O plano recomendou ações induzidas e a formulação de programas estratégicos visando à redução dessas disparidades e à melhoria da qualidade da pós-graduação brasileira de forma equânime entre as regiões e entre os estados. As ações e os programas voltados para as áreas de biotecnologia, nanotecnologia, fármacos, microeletrônica, biodiversidade, energia e áreas relacionadas à exploração do mar e ao desenvolvimento da região amazônica, entre outras, foram priorizados (BRASIL, 2005c).

Tendo por base os estudos realizados para elaboração do PNPG 2011-2020, verificou-se que, apesar das várias ações induzidas desenvolvidas pela Capes em atendimento às recomendações dos Planos Nacionais de Pós-Graduação anteriores, continuava sendo necessária tal atuação em diversas áreas e linhas de pesquisa. Assim, a ação indutiva da Capes e de outras agências de fomento tornou-se cada vez mais presente (BRASIL, 2010).

Em razão das bruscas alterações ocorridas no sistema científico e tecnológico, do alto custo gerado pelas atividades de pós-graduação e da consequente necessidade de mudança de rumo das políticas de educação, ciência e tecnologia, ganharam destaque também as ações voltadas para o incentivo à formação de redes cooperativas de pesquisa, como está dito em Rocha-Neto (2010). Essas redes foram contempladas inicialmente pelo III Plano Nacional de Pós-Graduação, que estabeleceu como estratégia "estimular formas de cooperação entre programas de pós-graduação, incluindo intercâmbio de pesquisadores e estudantes, uso comum de equipamentos e realização de pesquisa interdisciplinar e/ou multi-institucional” (BRASIL, 2005b, p. 210). Os planos nacionais de pós-graduação seguintes, especialmente o PNPG 2005-2010, reforçaram a importância das redes cooperativas como ferramentas 
para a melhoria da qualidade da pós-graduação e para a redução das assimetrias regionais (BRASIL, 2005c).

No entender de Lara e Lima (2009, p. 618), "colaboração científica", tal como apresentado na obra Redes sociais e colaborativas em informação científica, é um "processo social intrínseco às formas de interação humana para efetivar a comunicação e o compartilhamento de competências e recursos. Um meio para otimizar recursos, dividir o trabalho e criar sinergia entre os membros da equipe [...]".

De acordo com Balancieri et al. (2005), a colaboração científica pode ser considerada como um empreendimento cooperativo, que abrange metas comuns, esforço coordenado e resultados com responsabilidade e mérito compartilhados. Enfatizam ainda os autores que estudos realizados na década de 1980 a respeito da colaboração, sob a ótica dos resultados dos trabalhos científicos, comprovaram que o impacto é maior quando realizados por um grupo de pesquisadores.

Na concepção de Morosini e Franco (2001), a importância do trabalho em rede aumentou a partir da percepção de que a mobilidade associativa de produção é estratégica, pois possibilita a conquista do poder político na própria atividade científica e o reconhecimento da comunidade acadêmica.

Nesse contexto, a Capes criou vários programas com o intuito de incentivar a cooperação interinstitucional e reduzir os desequilíbrios regionais. Entre eles, o Programa Institucional de Capacitação Docente e Técnica PICDT, que induzia as universidades a planejarem suas necessidades de capacitação do corpo docente, envolvendo a instituição de origem de vínculo empregatício do docente e a instituição que oferecia o curso de pós-graduação, como ressaltado por Moura (2001). Outros programas instituídos com a mesma finalidade, o mestrado interinstitucional (Minter) e o doutorado interinstitucional (Dinter), possibilitam o oferecimento de uma turma de mestrado ou de doutorado fora da sua sede, por um período determinado, para formação de mestres e doutores nas suas instituições de origem, geralmente localizadas fora dos grandes centros (BRASIL, 2015). Entretanto, o Procad, um 
dos programas que substituiu o PICDT, é a principal ação da Capes voltada à redução das disparidades regionais na pós-graduação e ao estabelecimento de parcerias entre as instituições (CAPES, 2010).

Conforme diretrizes constantes no Plano Nacional de PósGraduação 2011-2020, o Procad deverá ter continuidade e será necessário instituir uma sistemática de acompanhamento e avaliação do programa (BRASIL, 2010). Embora o Procad tenha sido criado no ano 2000, sua sistemática atual só permite que seja realizada a avaliação das atividades desenvolvidas por cada projeto após dois anos de vigência, a fim de verificar se as metas prometidas nos dois primeiros anos foram executadas e decidir sobre a renovação ou não do financiamento.

Este artigo teve como propósito apresentar e analisar as percepções, as críticas e as sugestões dos consultores que participaram de processos avaliativos, no âmbito do Programa Nacional de Cooperação Acadêmica, tanto na submissão de propostas quanto na avaliação intermediária dos projetos em andamento, visando contribuir para o aprimoramento do programa.

\section{Metodologia}

A metodologia utilizada é de natureza qualitativa e quantitativa, considerando o fato de que, de acordo com Minayo (2012), as duas abordagens são complementares. Caracteriza-se também como um estudo exploratório e descritivo. Utilizou-se a estatística descritiva para análise dos dados quantitativos e, para o estudo dos dados qualitativos, a análise de conteúdo, como abordado por Bardin (2011).

Elaborou-se um instrumento de consulta, que foi submetido à validação por um pequeno grupo de avaliadores do Procad, antes de sua aplicação, para verificação quanto à pertinência e à compreensão das questões. Após a validação, o instrumento foi enviado, no mês de agosto de 2013, por correio eletrônico, aos integrantes da Comissão de Avaliação de Projetos Procad das edições de 2005 a 2009, que participaram tanto de processos de avaliação de novos projetos para efeito de concessão de 
benefícios, quanto de avaliações intermediárias do programa. Não foram selecionados consultores que participaram de avaliações relacionadas às edições do Procad lançadas em 2000 e 2001, considerando que, nesse período, não havia uma comissão específica para avaliação dos projetos, bem como do caráter experimental dessas edições. O questionário foi composto por 15 quesitos de natureza qualitativa, contando com 13 questões fechadas e duas abertas, relacionadas aos objetivos e às características do Procad, com o intuito de avaliar o programa sob o ponto de vista dos consultores que participaram de processos avaliativos intermediários e para efeito de novas concessões. Foi utilizada a escala de Likert com algumas adaptações, contendo as seguintes opções de respostas: (0) "não sei", (1) "discordo totalmente", (2) "discordo parcialmente", (3) "concordo parcialmente" e (4) "concordo totalmente".

\section{O Procad: antecedentes e estrutura atual}

Em atendimento às recomendações dos Planos Nacionais de Pós-Graduação e considerando a necessidade de implementar ações que suprissem a carência de instrumentos de apoio à formação de docentes do ensino superior, ocasionada pela extinção do PICDT, foi lançado, no ano de 2000, o primeiro edital do Procad, como projeto-piloto. Por meio do programa, seria possível promover a qualificação do corpo docente do ensino superior vinculada a projetos de pesquisa institucionais, de forma integrada e cooperativa, bem como elevar de forma mais equilibrada os niveis de qualidade da pós-graduação brasileira (BRASIL, 2005a).

O Procad possibilita a utilização de recursos humanos e da infraestrutura das instituições de ensino superior para a abordagem de novos tópicos de pesquisa e a criação de condições estimulantes à associação de projetos para o fortalecimento da produção de conhecimentos e da formação pós-graduada. Os programas de pósgraduação considerados consolidados, ou seja, avaliados pela Capes com nota igual ou superior a 5, associam-se a outros programas ainda em fase de consolidação, geralmente cursos novos avaliados com notas 3 e 4, ou fazem associações entre programas consolidados para apresentar projetos conjuntos, envolvendo a realização de missões de pesquisa e 
docência e de estudos. O programa financia passagens, diárias, bolsas de iniciação científica, doutorado e pós-doutorado e outras despesas de custeio. Além disso, apoia a mobilidade de estudantes, por meio da modalidade "bolsa sanduíche"1 no país e de auxílio-moradia, bem como a mobilidade de docentes dos programas de pós-graduação envolvidos no projeto (BRASIL, 2005a).

Vieram corroborar a pertinência do Procad as diretrizes do PNPG 20052010, que determinam o seguinte:

\footnotetext{
Diante da existência de um quadro de assimetrias, torna-se necessário que o PNPG 2005-2010 contemple a indução de programas, como linha programática, visando reduzir as diferenças regionais, intra-regionais e entre Estados, bem como estabelecer programas estratégicos buscando a sua integração com políticas públicas de médio e longo prazos (BRASIL, 2005c, p. 59)
}

Com base nessas recomendações, o Procad ganhou reforço como meio para a melhoria da qualidade do Sistema Nacional de PósGraduação e novas linhas de atuação, tais como o Programa Nacional de Cooperação Acadêmica Amazônia - Procad-Amazônia e o Programa Nacional de Cooperação Acadêmica Novas Fronteiras - Procad-NF, voltados, especialmente, à redução das assimetrias regionais (CAPES, 2010).

O Procad-Amazônia foi lançado em 2006, com o objetivo de apoiar projetos conjuntos de pesquisa realizados por instituições de ensino superior pertencentes à região amazônica em associação com outras instituições do país, proporcionando a ampliação da formação de mestres e doutores e da produção científica, o intercâmbio docente e discente, a criação de novos programas de pós-graduação e a fixação de doutores na Amazônia brasileira (BRASIL, 2006).

Trata-se de bolsa dirigida aos discentes de mestrado e doutorado para realização de estágio de um a 12 meses em uma das instituições integrantes da parceria. 
A Capes lançou, até o momento, nove editais do Procad. O programa, em âmbito nacional, contemplou cinco edições, instituídas em 2000, 2001, 2005, 2007 e 2013. O Procad-Amazônia contou com apenas um edital, lançado em 2006. O Procad-NF teve três edições, em 2007, 2008 e 2009. O financiamento total dessas edições foi da ordem de R $\$$ 268.018.834,00. Foram apoiados, no total, 903 projetos, abrangendo 2.301 equipes vinculadas a diversos programas de pós-graduação e áreas do conhecimento. Foi concedido o total de 9.480 bolsas, nos niveis de iniciação científica, mestrado sanduíche, doutorado sanduíche, doutorado pleno e pós-doutorado, de acordo com Moura e Rocha-Neto (2015). No Quadro 1, a seguir, são especificados tais dados, por edição do programa.

\begin{tabular}{|l|r|r|r|r|}
\hline \multicolumn{1}{|c|}{ Edital } & $\begin{array}{r}\text { Projetos } \\
\text { apoiados }\end{array}$ & Equipes & $\begin{array}{l}\text { Bolsas } \\
\text { concedidas }\end{array}$ & \multicolumn{1}{c|}{$\begin{array}{c}\text { Financiamento } \\
\text { (R\$) }\end{array}$} \\
\hline Procad 2000 & 33 & 78 & 506 & $7.188 .770,76$ \\
\hline Procad 2001 & 85 & 217 & 903 & $19.496 .197,00$ \\
\hline Procad 2005 & 132 & 337 & 1.017 & $32.204 .045,43$ \\
\hline Procad 2007 & 207 & 563 & 994 & $49.431 .989,00$ \\
\hline Procad 2013 & 100 & 313 & 3.752 & $77.314 .815,00$ \\
\hline $\begin{array}{l}\text { Procad -Amazônia } \\
\text { 2006 }\end{array}$ & 28 & 56 & 63 & $6.241 .898,32$ \\
\hline Procad-NF 2007 & 82 & 185 & 271 & $19.186 .812,00$ \\
\hline Procad-NF 2008 & 110 & 227 & 421 & $22.015 .709,49$ \\
\hline Procad-NF 2009 & 126 & 325 & 1.553 & $34.938 .597,00$ \\
\hline \multicolumn{1}{|c|}{ Total geral } & 903 & $\mathbf{2 . 3 0 1}$ & $\mathbf{9 . 4 8 0}$ & $\mathbf{2 6 8 . 0 1 8 . 8 3 4 , 0 0}$ \\
\hline
\end{tabular}

Quadro 1. Investimentos Procad (2000 a 2013)

Fonte: elaboração dos autores.

\section{Resultados e discussões}

Com relação ao instrumento de consulta utilizado, foram respondidos 13 questionários, o que corresponde a 100\% do total enviado. Cabe destacar que todas as questões contempladas pelo questionário foram respondidas na sua totalidade por todos os 
avaliadores. Pode-se compreender que a participação de todos os avaliadores de projetos Procad consultados ocorreu provavelmente devido ao seu reconhecimento da importância de tal política pública e, consequentemente, ao interesse em contribuir com a pesquisa. A seguir, são apresentados nas Figuras de 1 a 13, os resultados referentes às questões fechadas da pesquisa de opinião aplicada aos avaliadores de projetos Procad.

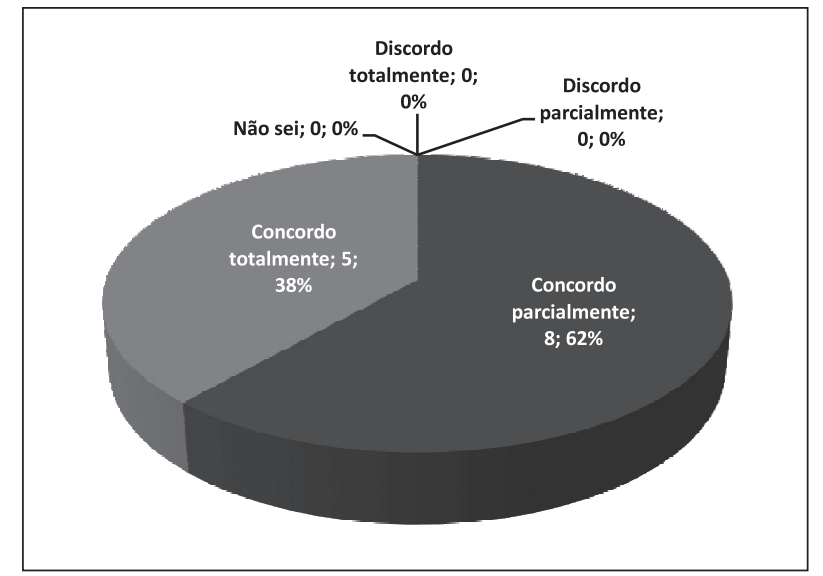

Figura 1. 0 Procad tem cumprido suas metas

Fonte: elaboração dos autores com base na pesquisa com avaliadores.

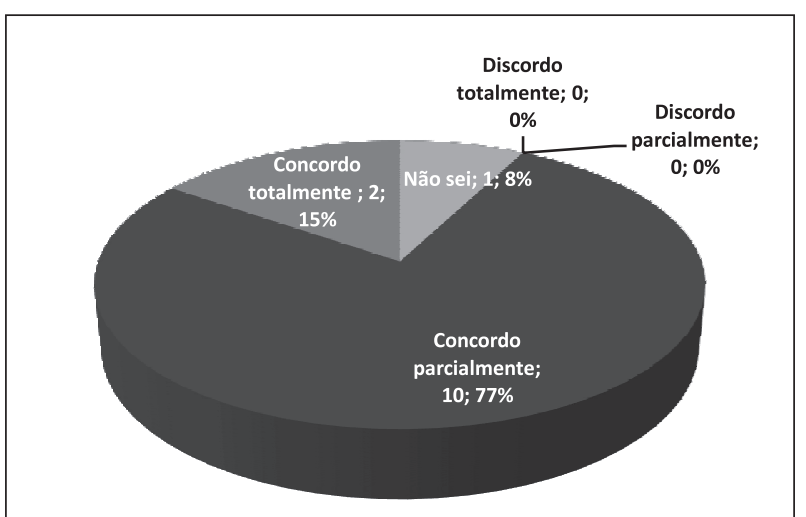

Figura 2. As missões de pesquisa estão sendo realizadas conforme previsto nos projetos

Fonte: elaboração dos autores com base na pesquisa com avaliadores. 


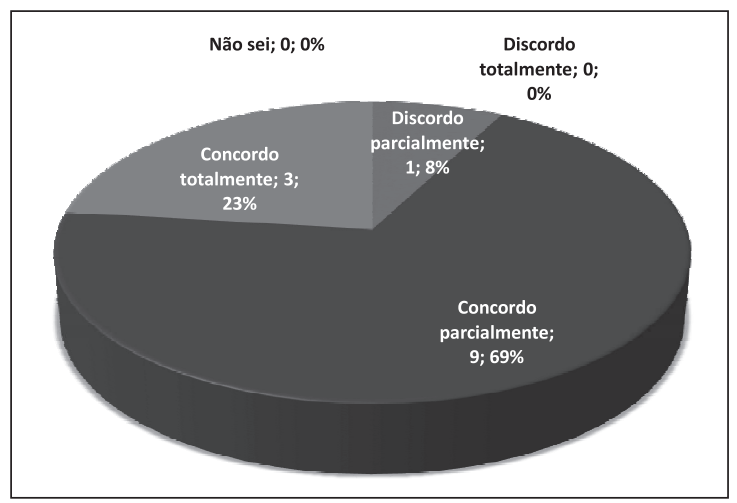

Figura 3. As missões de docência estão sendo realizadas conforme previsto nos projetos

Fonte: elaboração dos autores com base na pesquisa com avaliadores.

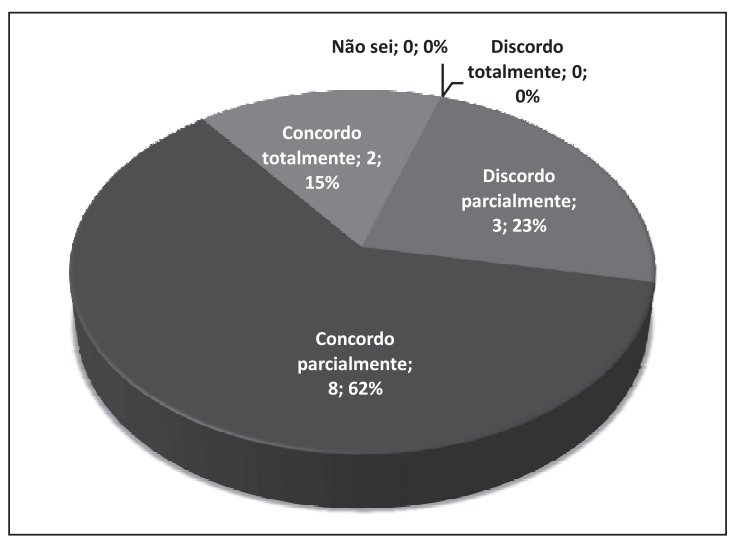

Figura 4. As missões de estudos estão sendo realizadas conforme previsto nos projetos

Fonte: elaboração dos autores com base na pesquisa com avaliadores.

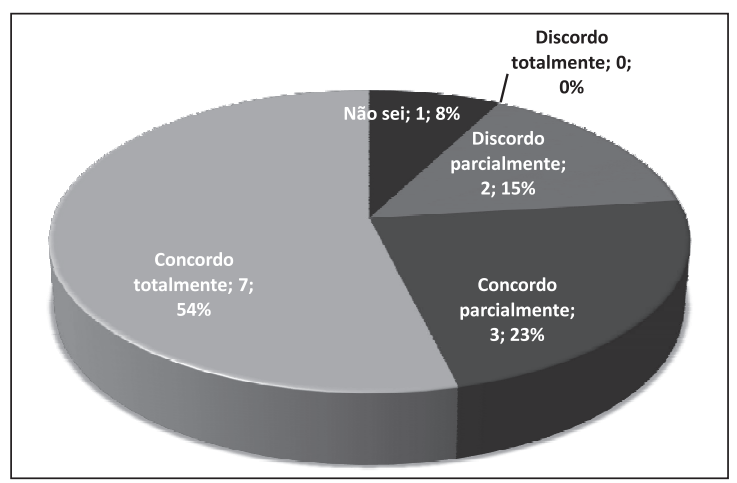

Figura 5. A duração das missões de pesquisa tem atendido às necessidades dos projetos

Fonte: elaboração dos autores com base na pesquisa com avaliadores. 


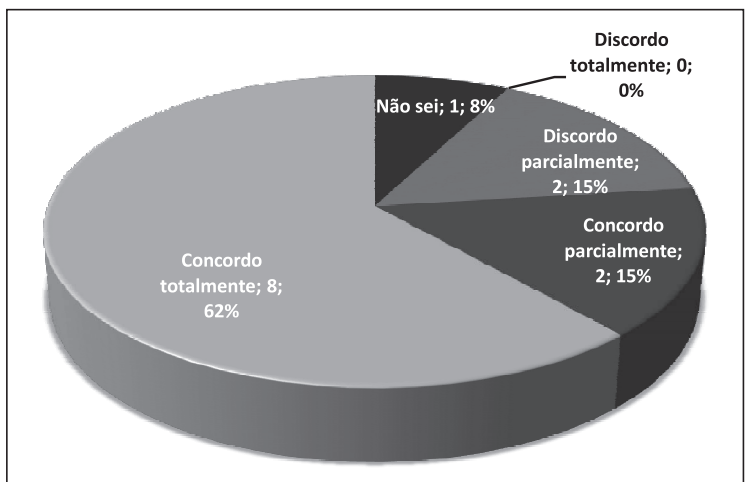

Figura 6. A duração das missões de docência tem atendido às necessidades dos projetos

Fonte: elaboração dos autores com base na pesquisa com avaliadores

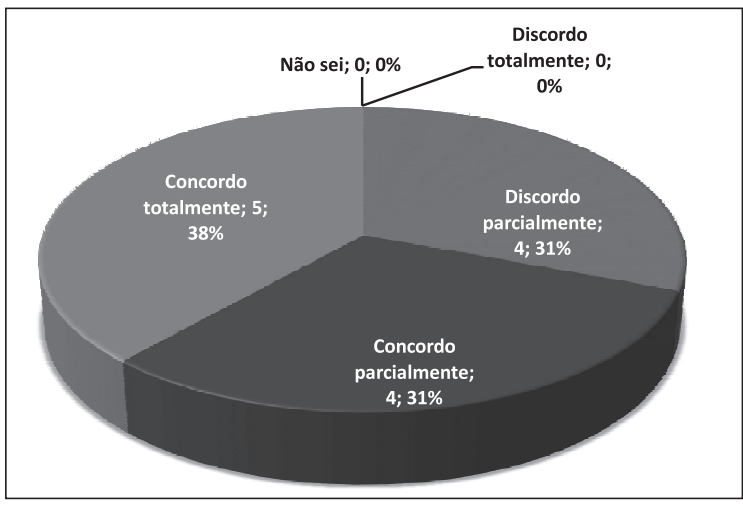

Figura 7. A duração das missões de estudo tem atendido às necessidades dos projetos

Fonte: elaboração dos autores com base na pesquisa com avaliadores.

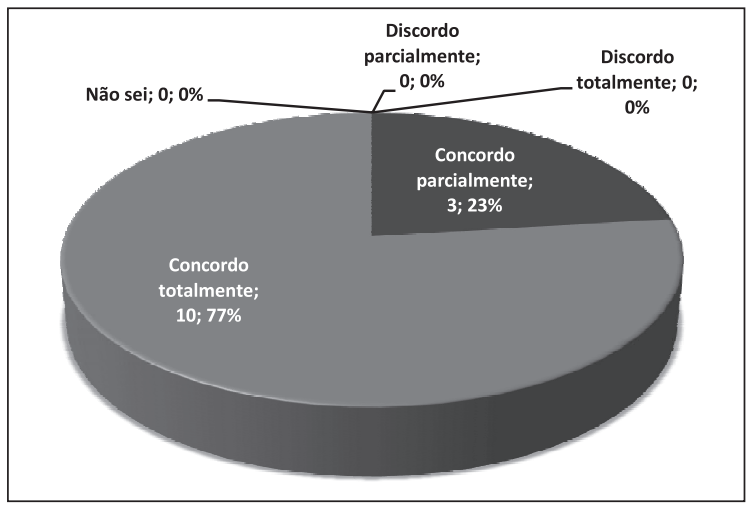

Figura 8. As missões têm contribuído para o fortalecimento da cooperação entre as equipes participantes

Fonte: elaboração dos autores com base na pesquisa com avaliadores. 


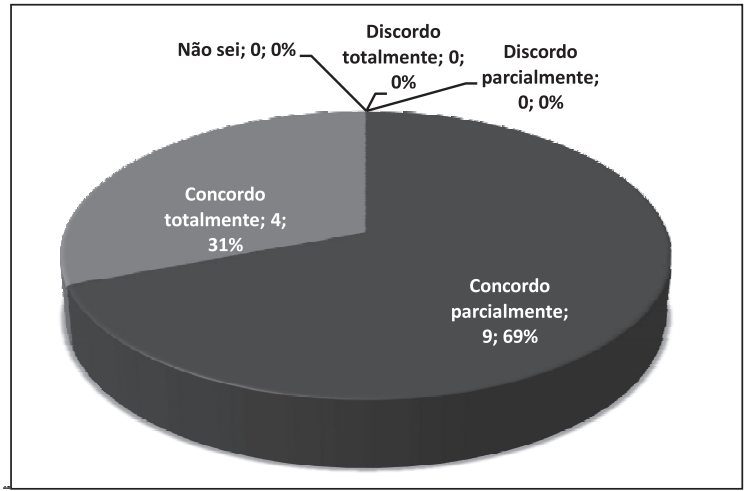

Figura 9. Os projetos têm contribuído para o aumento da produção científica conjunta dos programas cooperantes

Fonte: elaboração dos autores com base na pesquisa com avaliadores.

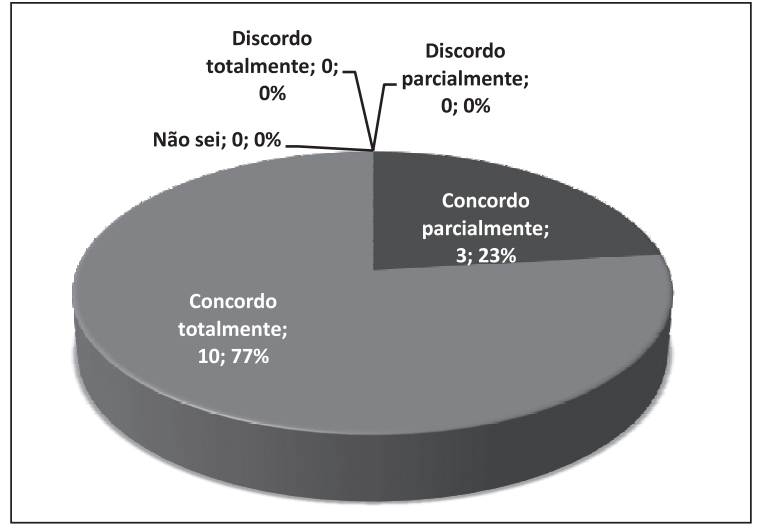

Figura 10. Os projetos têm sido eficazes para a qualificação de pessoal

Fonte: elaboração dos autores com base na pesquisa com avaliadores.

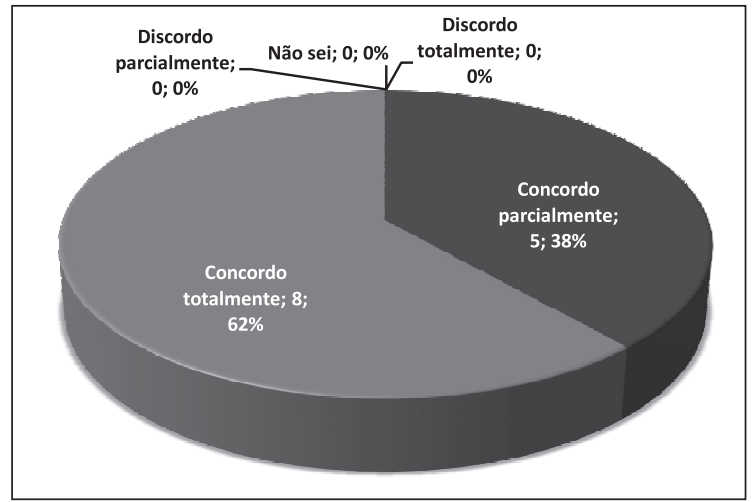

Figura 11. 0 programa está contribuindo para a diminuição da desigualdade regional na pós-graduação brasileira

Fonte: elaboração dos autores com base na pesquisa com avaliadores. 


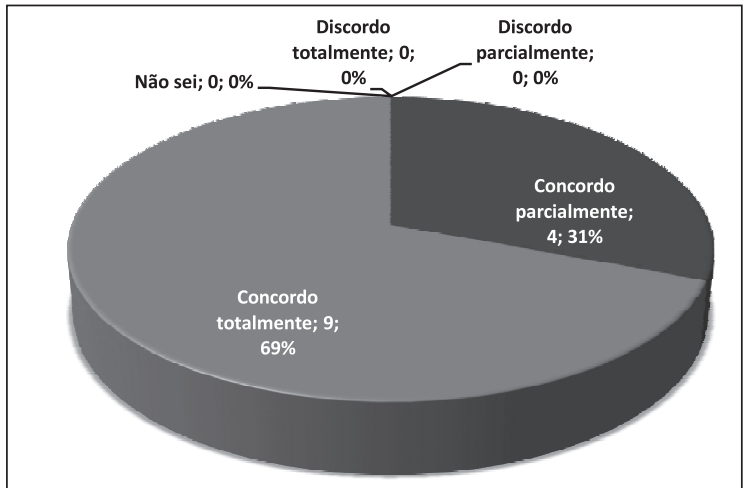

Figura 12. Os recursos financeiros têm sido suficientes para o alcance das metas

Fonte: elaboração dos autores com base na pesquisa com avaliadores.

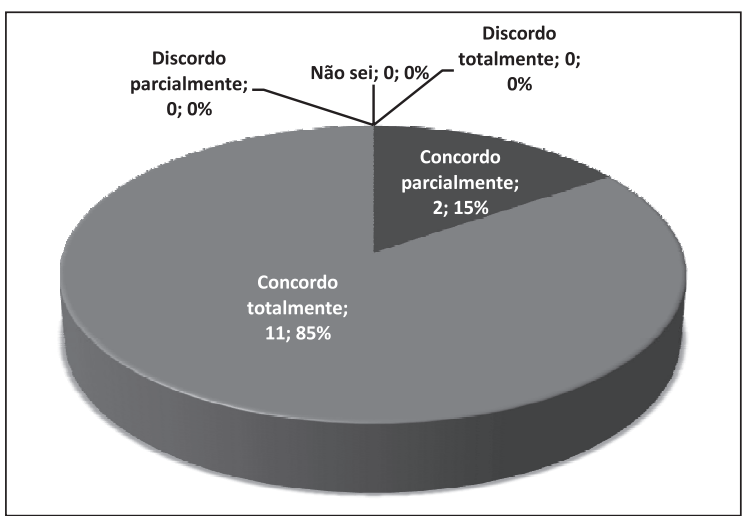

Figura 13. A gestão do Programa tem sido feita de forma ágil e flexível

Fonte: elaboração dos autores com base na pesquisa com avaliadores.

Verificou-se que o maior número de respostas relativas às questões fechadas do questionário ficou entre as opções "concordo totalmente" (50\%) e "concordo parcialmente" (41\%), totalizando $91 \%$. Conforme se pode verificar nas Figuras 1, 8, 9, 10, 11, 12 e 13, essas duas opções representaram 100\% das respostas obtidas. Em estudo, no qual foi utilizado instrumento de consulta semelhante, elaborado com base no ponto de vista dos coordenadores de projetos Procad, o total de respostas "concordo totalmente" e "concordo parcialmente" foi de 79\%, como se vê em Moura e Rocha-Neto (2015). Compreende-se, com tais resultados, que o Procad tem atingido os principais objetivos que orientaram a criação do programa. 
Os resultados demonstraram ainda que a grande maioria reconhece que a gestão do programa tem sido feita de forma ágil e flexível, pois essa afirmativa recebeu o maior número de respostas com a opção "concordo totalmente" (85\%). Entretanto, considerando que 15\% das respostas atribuídas a essa afirmação ficaram com concordância parcial, ainda há indicação de necessidade de melhorias com relação a esse aspecto.

Os resultados relativos às afirmações constantes das Figuras 2 e 3, que tratam da execução das missões de pesquisa e docência, de acordo com a previsão do projeto, apresentaram um pequeno grau de incerteza e discordância. Por outro lado, a afirmação referente à Figura 4, que aborda o mesmo tópico relacionado à missão de estudo, teve uma quantidade maior de discordância em relação aos quesitos relativos às Figuras 2 e 3, merecendo maior atenção por parte da Capes.

Constatou-se que as afirmações referentes às Figuras 5 e 6, que tratam da duração das missões de pesquisa e de docência, tiveram resultados que demonstram que grande parte dos consultados concorda em que essas missões estão condizentes com as necessidades dos projetos, entretanto, também há um certo grau de incerteza e de discordância com relação a esse aspecto. Por outro lado, a afirmativa relativa à Figura 7, que aborda o mesmo tema concernente às missões de estudo, apresentou resultados que indicam maior grau de discordância em relação aos demais quesitos do questionário, o que requer um novo olhar sobre o prazo de duração das missões de estudo estipulado pelo programa.

Tendo por base as respostas às questões abertas do questionário aplicado aos avaliadores de projetos Procad, na sequência de cada pergunta, foram listados os principais benefícios e sugestões relacionados ao programa, segundo os avaliadores consultados.

Em relação à pergunta "Do seu ponto de vista, quais os benefícios que os projetos Procad têm ensejado aos programas de pós-graduação participantes?", foram obtidos como resposta os seguintes benefícios: 
a) a mobilidade nacional entre discentes e docentes de programas de pós-graduação de diferentes regiões resultou em grande avanço e trouxe possibilidades que antes não existiam, inovando nesse aspecto;

b) a oportunidade de desenvolver, realizar e publicar trabalhos em colaboração com pesquisadores de outros centros pode ser um diferencial importante e decisivo para um grupo de pesquisadores de um programa de pós-graduação em consolidação ou em vias de afirmação em alguma área de estudo;

c) a possibilidade de obtenção de recursos para diferentes atividades (bolsas, passagens, diárias, verba de custeio etc.);

d) a permissão para a utilização dos recursos alocados (exceto bolsas) diretamente pelo coordenador, com um mínimo de burocracia;

e) a duração do projeto ( 4 anos) permite a solidificação de parcerias, inclusive o engajamento de estudantes sem a preocupação com a falta de recursos materiais para a efetiva participação dos mestrandos e doutorandos;

f) o estabelecimento de parcerias em projetos de cooperação, que vêm contribuindo para a consolidação dos programas de pós-graduação participantes, particularmente daqueles situados no Norte, Nordeste e Centro-Oeste;

g) a contribuição para a formação de graduados, mestres e doutores de alto nível e ampliação da produção científica em revistas indexadas;

h) a possibilidade e o enfrentamento das desigualdades regionais relacionadas à pesquisa e à pós-graduação no Brasil;

i) o fato de que nos programas com apenas mestrado, alguns alunos inseridos no Procad dão sequência no curso de doutorado nas instituições parceiras, garantindo assim a longevidade da cooperação iniciada.

E, quanto à segunda questão, "Quais são as suas sugestões para o aperfeiçoamento da gestão do Procad?”, as ideias apresentadas foram: 
a) acompanhar a execução dos recursos alocados, especialmente nos casos em que os percentuais de utilização sejam extremamente baixos ou em que a realização dos gastos ocorram na data limite, e as equipes que, ao final de dois anos, não tenham utilizado pelo menos $60 \%$ dos recursos disponiveis deveriam ter os projetos encerrados;

b) custear também itens de capital, pois são necessários para a melhoria dos programas e para a obtenção de livros, computadores etc.;

c) flexibilizar a duração das missões de estudo e aumentar o custeio dessas missões, priorizando o financiamento das missões de média e longa duração;

d) realizar reuniões iniciais com as equipes coordenadora e associada, para detalhamento de todas as etapas do projeto, bem como visitas a algumas equipes com dificuldades de implementação dos projetos e/ ou que demonstrem interesse;

e) utilizar um sistema de certificação da produção científica compartilhada entre os grupos envolvidos, pois, nos relatórios apresentados, observa-se que muitos pesquisadores ainda relacionam a produção individual como fruto da parceria;

f) buscar uma forma de equilibrar a efetivação das missões de pesquisa e docência e de estudo entre os grupos de pesquisa, pois a maioria dos pesquisadores das IES que possuem 0 programa de nível mais elevado são os que realizam o maior número de missões de docência e de pesquisa, enquanto que o inverso ocorre em relação aos alunos;

g) possibilitar a adequação das metas durante o andamento do projeto, sem que haja prejuízo em relação aos objetivos propostos, considerando o fato de que, mesmo que a gestão do programa pela Capes tenha sido muito eficaz, a maioria dos coordenadores dos projetos ainda não foi capaz de gerenciálos com a eficiência desejada;

h) liberar as bolsas de mobilidade por cotas para as pró-reitorias, da mesma forma que o Programa de Doutorado Sanduíche com Estágio no Exterior (PDSE), da Diretoria de Relações Exteriores (DRI/Capes), facilitando a mobilidade dos alunos de diversos programas dentro do Brasil; 
i) estabelecer a exigência de inserção dos relatórios de acompanhamento no Coleta Capes (via Sucupira), uma vez que o coordenador teria de elencar, entre a produção científica do programa, as oriundas do Procad, assim como as defesas de trabalhos finais, seminários, workshops etc., garantindo, inclusive, melhor controle dos intercâmbios e ações realizadas;

j) realizar projetos Procad preferencialmente com os coordenadores dos programas emergentes na coordenação do intercâmbio discente, possibilitando o intercâmbio com um número maior de opões de destino, de temas e de laboratórios, bem como permitir a realização de missões discentes também no exterior;

k) viabilizar projetos Procad em áreas que ainda não apresentam programas de pós-graduação com notas 6 e 7, como é o caso da área de Recursos Florestais/Engenharia Florestal, representada por programas localizados em diferentes biomas brasileiros.

Com base nas respostas relativas aos benefícios do Procad, é possivel afirmar que todos os respondentes reconhecem que o programa tem incentivado a constituição de redes de pesquisa, a mobilidade docente e discente, o aumento da formação de pessoal qualificado e da produção de conhecimentos, além de contribuir para a redução das assimetrias regionais na pós-graduação brasileira. Resultados semelhantes foram encontrados em pesquisa de Moura e RochaNeto (2015) sobre o mesmo tema, abordando o ponto de vista dos coordenadores de projetos Procad.

Verificou-se também que há consenso entre os respondentes desta pesquisa e os coordenadores de projeto Procad participantes de estudo realizado por Moura e Rocha-Neto (2015) em algumas das sugestões apresentadas, tais como o financiamento de itens de capital, a flexibilização das missões de estudo e das metas estabelecidas no projeto e a realização de reuniões com os coordenadores de projetos Procad. Por outro lado, observou-se que houve maior preocupação por parte dos respondentes da pesquisa com relação à necessidade de 
instituição de mecanismos de acompanhamento do programa com o objetivo de tornar mais eficiente a gestão dos recursos financeiros por parte dos beneficiários de projetos Procad e mais transparentes os resultados alcançados por meio do programa.

Em outro artigo, abordando tema semelhante e abrangendo apenas a edição Procad-NF 2007, Gomes e Rocha-Neto (2011), confirmaram a importância do Procad como instrumento de política pública que tem favorecido a qualificação de pessoal de alto nível por intermédio de redes de pesquisa e a diminuição do desequilíbrio regional na pós-graduação, bem como o crescimento do potencial de competitividade do país.

\section{Considerações finais}

Verificou-se que a principal preocupação dos participantes da pesquisa está relacionada à necessidade de instituição de processos de acompanhamento e avaliação do Procad. Nesse sentido, a Capes poderia utilizar o Sistema de Prestação de Contas on-line-Siprec. O relatório de execução das atividades, de periodicidade anual, e o relatório final, que já constam do sistema, poderiam ser adaptados para contemplar também informações necessárias à avaliação intermediária e final de cada projeto. Após o término da vigência de cada edição do programa, poderia ser realizada uma avaliação geral, para verificar se foram alcançados os objetivos propostos, bem como para sugerir aperfeiçoamentos para as edições futuras.

Finalmente, com base neste estudo, pode-se compreender que a institucionalização de um sistema de avaliação contínua para o Procad poderia contribuir para o aprimoramento do programa e para a criação de novas ações voltadas ao desenvolvimento da pós-graduação. A implantação de medidas aqui sugeridas também proporcionaria melhorias ao Procad.

Recebido em 03/11/2015

Aprovado em 16/02/2016 


\section{Referências}

BALANCIERI, R. et al. A análise de redes de colaboração científica sob as novas tecnologias de informação e comunicação: um estudo na Plataforma Lattes. Ciência da Informação, v. 34, n. 1, p. 64-77, 2005.

BARDIN, L. Análise de conteúdo. Tradução de Luís Antero Reto e Augusto Pinheiro. São Paulo: Edições 70, 2011.

BRASIL. Instruções para apresentação de projetos do programa nacional de cooperação acadêmica: edital PROCAD n 01/2005. Brasília: Capes, 2005a.

BRASIL. Ministério da Educação. Coordenação de Aperfeiçoamento de Pessoal de Nivel Superior. Instruções para apresentação de projetos do programa nacional de cooperação acadêmica Amazônia: edital PROCAD nº 01/2006. Brasília: Capes, 2006.

Plano Nacional de Pós-Graduação: PNPG 2005-2010. Brasília: Capes, 2005b. 262p.

Plano Nacional de Pós-Graduação: PNPG 2005-2010. Brasília: Capes, 2005c, 262p.

Plano Nacional de Pós-Graduação: PNPG 2011-2020. Brasília: Capes, 2010. v. 1. Disponivel em: <http://www.capes.gov.br/images/ stories/download/Livros-PNPG-Volume-I-Mont.pdf>. Acesso em: 15 ago. 2014

Projetos de mestrado e doutorado interinstitucionais - MINTER/ DINTER nacionais e internacionais: edital n 11/2015. Brasília: Capes, 2015.

COORDENAÇÃO DE APERFEIÇOAMENTO DE PESSOAL DE NIVELL SUPERIOR - CAPES. Relatório de gestão da CAPES: 2004-2010. Brasília: Capes, 2010. 
GOMES, D. B. B. A.; ROCHA-NETO, I. Avaliação de progresso do programa nacional de cooperação acadêmica - novas fronteiras - edição 2007 e oportunidades de formação de redes cooperativas nas áreas estratégicas e tecnologias portadoras de futuro. Revista Brasileira de Educação, Brasília, v. 16, n. 48, 2011. Disponivel em: <www.scielo.br/pdf/rbedu/ v16n48/v16n48a10.pdf>. Acesso em: 15 ago. 2014.

GUIMARÃES, J. A. A nova agenda da sociedade brasileira. Gestor CT\&I: ciência, tecnologia e inovação - Consecti, Brasília, ano 2, n. 3, 2009. Disponível em: <http://www.consecti.org.br/wp-content/ uploads/2010/02/revista-gestor-cti-03.pdf>. Acesso em: ago. 2015.

LARA, M. L. G.; LIMA, V. M. A. Termos e conceitos sobre redes sociais. In: POBLACIÓN, D. A.; MUGNANI, R.; RAMOS, L. M. S. V. C. (Org.). Redes sociais e colaborativas em informação científica. São Paulo: Angellara, 2009.

MINAYO, M. C. Souza (Org.). Pesquisa social: teoria, método e criatividade. 31. ed. Petrópolis, RJ: Vozes, 2012.

MOROSINI, M. C.; FRANCO, M. E. D. P. Gestão de redes de pesquisa em Educação Superior: questão de princípios e ethos. In: FRANCO, M. E. D. P; MOROSINI, M. C. (Org.). Redes acadêmicas e produção do conhecimento em educação superior. Brasília: Instituto Nacional de Estudos e Pesquisas Educacionais, 2001.

MOURA, E. V. Limites e perspectivas do programa institucional de capacitação docente e técnica - PICDT. 2001. Dissertação (Mestrado em Políticas e Gestão de Ciência e Tecnologia)-Centro de Desenvolvimento Sustentável, Universidade de Brasília, Brasília, 2001.

MOURA, E. V.; ROCHA-NETO, I. Avaliação do Programa Nacional de Cooperação Acadêmica - PROCAD. Enciclopédia Biosfera, Centro Científico Conhecer, Goiânia, v. 11, n. 22, p. 3.502-3.518, 2015.

ROCHA-NETO, I. Prospectiva da pós-graduação no Brasil (2008-2022). RBPG, Brasília, v. 7, n. 12, p. 58-79, 2010. Disponível em: <http://ojs. rbpg.capes.gov.br/index.php/rbpg/article/view/181/175>. Acesso em: ago. 2015. 